\title{
Clinical profile and outcome of scar endometriosis in a tertiary care centre of Northern India
}

\author{
Sunil Kumar Juneja, Pooja Tandon*, Anshu Sharma, Anjali Sharma
}

Department of Obstetrics and Gynecology, DMCH, Ludhiana, Punjab, India

Received: 01 July 2019

Revised: 23 December 2019

Accepted: 27 December 2019

\author{
*Correspondence: \\ Dr. Pooja Tandon, \\ E-mail: drpoojatandon77@gmail.com
}

Copyright: (c) the author(s), publisher and licensee Medip Academy. This is an open-access article distributed under the terms of the Creative Commons Attribution Non-Commercial License, which permits unrestricted non-commercial use, distribution, and reproduction in any medium, provided the original work is properly cited.

\begin{abstract}
Background: Endometriosis is defined as the presence and proliferation of the endometrium outside the uterine cavity affecting an estimated 89 million women of reproductive age worldwide. Endometriosis occurs in 5\% to $10 \%$ of all women, often resulting in debilitating pain and infertility, endometriosis at the scar site known as Scar endometriosis has also been described following obstetrical and gynecological surgery. Scar endometriosis has a much rarer incidence with an illusive presentation and is often misdiagnosed and definitive treatment gets delayed. Objectives of this study was to review the demographic profile, clinical presentation, treatment modalities and outcome in patients with scar endometriosis.

Methods: The retrospective study of patients with scar endometriosis managed at DMCH during January 2014 to December 2018 was done.

Results: Eight patients of scar endometrosis were diagnosed and operated in our institution over a period of five years. All patients (six with previous caesarean section scar site and two at episiotomy site) presented with tender nodules with pain which got exaggerated during menstruation.

Conclusions: A high index of suspicion of scar endometriosis should be kept in patients presented with cyclical pain and tender mass getting exaggerated during menstruation.
\end{abstract}

Keywords: Cyclical pain, Scar endometriosis, Tender mass

\section{INTRODUCTION}

Endometriosis is defined as the presence and proliferation of the endometrium outside the uterine cavity. Affecting an estimated 89 million women of reproductive age worldwide, endometriosis occurs in $5 \%$ to $10 \%$ of all women, often resulting in debilitating pain and infertility. ${ }^{1}$ It mainly affects women in reproductive ages. It is seen in $1-2 \%$ of women undergoing sterilization or sterilization reversal, in $10 \%$ of hysterectomy surgeries, in $16-31 \%$ of laparoscopies, and $53 \%$ of adolescents with pelvic pain severe enough to warrant surgical evaluation. There are two types of endometriosis: internal endometriosis (adenomyosis and myometrial adenomyosis) and external endometriosis: genital (located on the genitals or pelvic ligaments) and extragenital (intestine, lung, pleura, kidneys, surgical scars localizations, etc). ${ }^{2-5}$ Most often endometriosis occur in pelvis but many times the surface lining of pelvic cavity, peritoneum, ovaries, posterior cul-de-sac, and uterosacral ligaments are involved too. Rarely, implants can occur outside of the pelvis and are termed as extra pelvic endometriosis. A rare case of cutaneous endometriosis has also been reported. It is observed that endometrioma can occur in the umbilicus even without predecessor surgery. 
Endometriosis at the scar site known as Scar endometriosis has also been described following obstetrical and gynecological surgery. Scar endometriosis has a much rarer incidence with an illusive presentation and is often misdiagnosed and definitive treatment gets delayed. We aimed to review the demographic profile, clinical presentation, treatment modalities and outcome in patients with scar endometriosis.

\section{METHODS}

A retrospective study was conducted at department of obstetrics and gynecology, DMCH over a period of 5 years during January 2014 to December 2018 to review the demographic profile, clinical presentation, treatment modalities and outcome in patient with scar endometriosis.

The histories, examination findings, investigations, treatment received in all these patients were reviewed. Inclusion criteria: all patients with FNAC proven scar endometrisis were included in the study. Patients presenting with cyclical pain and nodule at previous surgery site who did not undergo FNAC were excluded from the study. Data were described in terms of range; frequencies (number of cases) and relative frequencies (percentages) as appropriate.

\section{Statistical analysis}

All statistical calculations were done using SPSS (statistical package for the social science) SPSS 17 version statistical program for Microsoft Windows.

\section{RESULTS}

In our study we observed that seventy five percent of the patients were multiparous women (Table 1).

Table 1: Distribution of patients according to parity.

\begin{tabular}{|ll|}
\hline Sr. No. & Obstetrical formula \\
\hline 1. & P $1+1$ \\
\hline 2. & P $3+1$ \\
\hline 3. & P $1+0$ \\
\hline 4. & P $2+1$ \\
\hline 5. & P $2+2$ \\
\hline 6. & P2L2 \\
\hline 7. & P2L1A 1 \\
\hline 8. & P $3+1$ \\
\hline
\end{tabular}

Table 2: Clinical presentation.

\begin{tabular}{|lll|}
\hline Sr. No. & Symptoms and signs & \\
\hline 1. & Continuous pain at scar site & 2 \\
\hline 2. & $\begin{array}{l}\text { Cyclical pain at scar site } \\
\text { exaggerated during menstruation }\end{array}$ & 6 \\
\hline 3. & Tender mass/ nodule & 8 \\
\hline
\end{tabular}

The most important symptoms of scar endometriosis are presence of tender mass or nodule with cyclical pain at scar site exaggerated during menstruation (Table 2). The perineal scar endometriosis is associated with perineal mass and cyclical pain at previous episiotomy scar. The suspected diagnosis of endometriosis at the scar site was supported by the patient's history, which identified genital dysfunctions, a history of previous obstetrical surgery. The differential diagnosis of this entity includes suture granulomas, abscesses, hematomas, keloid, lipomas, sabeceous cysts, dermoids or malignant tumor. In our study majory of the patients had scar endometriosis following a cesarean section (Table 3 ). The interval between prior surgical treatment and onset of symptoms ranged from 1 to 20 years. The cases of endometriosis in the scars that we treated were diagnosed at 5, 7- and 12-years period of follow up after the initial surgery (Table 4).

Table 3: Site of scar endometriosis.

\begin{tabular}{|lll|}
\hline Sr. No. & Site of scar & No. of patients \\
\hline 1. & Vertical infraumblical & 4 \\
\hline 2. & Pfannsteil & 2 \\
\hline 3. & Episiotomy & 2 \\
\hline
\end{tabular}

Table 4: Time gap between previous surgery and definitive treatment.

\begin{tabular}{|lll|}
\hline Sr. No. & Time gap & No. of patients \\
\hline 1. & $>5$ years & 2 \\
\hline 2. & $5-10$ years & 5 \\
\hline 3. & $>10$ years & 1 \\
\hline
\end{tabular}

The use of imaging studies is indicated for surgical planning. The most reliable and accessible imaging test for it is ultrasound. It visualizes the parietal heterogeneous mass in the scar of abdominal wall. CTscan is not routinely indicated due to its poor imaging modality because of the lack of resolution and radiation exposure. MRI is currently the best imaging method to evaluate the extension of endometriosis lesions. The use of fine needle aspiration (FDA) helps in confirming the suspicious lesions as being endometriotic.

All our patients had received medical treatment prior to definitive surgery. Surgical treatment of the abdominal wall endometriosis required large extent of the excision to completely remove the lesion and prevent recurrence. One patient required treatment with mesh to support the rectus sheath. In all patients the excised tissue was sent for histopathological examination and the diagnosis of scar endometriosis was confirmed. Patients were followed up monthly for six months.

\section{DISCUSSION}

Endometriosis has been known for more than 300 years caused by functionally active ectopic endometrial areas 
located in the genital tract or adjacent pelvic structures. ${ }^{6}$ Scar endometriosis is a rare entity reported.

Endometriosis in abdominal incision sites are reported following cesarean sections, hysterectomy, ovarian endometrioma removal. Due to considerable increase of cesarean sections performed worldwide, it is probably in the rising trend. Its exact prevalence is unknown because it can be diagnosed only after surgery either open or laparoscopy. The incidence of endometriosis after cesarean section has been reported to be $0.03-0.4 \%$. Scar endometriosis patients are often referred to general surgeons because the clinical presentation suggests a surgical cause. Very few cases of endometriosis at episiotomy sites are also described in literature. Episiotomy scar endometriosis is a fairly rare site of endometriosis reported up to $1 \%$ of women following obstetrical procedures.

Scar endometriosis presents clinically as a painful, palpable subcutaneous mass with exaggeration during menses. A painful mass near scar with symptoms related to menstruation is pathognomonic of this condition, but only $20 \%$ of patients present with typical presentation. Therefore, it is often misdiagnosed as incisonal hernia, suture granuloma, lipoma, abscess, cyst hematoma or foreign body. Histopathological examination is the gold standard for diagnosis. Usually a classical triad of cyclical pain, perineal mass and previous episiotomy or tear is sufficient to clinch the diagnosis of episiotomy scar endometriosis. Sometimes patient presented with endometriosis nodules located at the episiotomy scar and involvement of the external anal sphincter.

Many theories have been postulated, the most generally accepted theory is the iatrogenic transplantation of endometrial implants to the wound edge during an abdominal or pelvic surgery. During cesarean section, endometrial tissue might be seeded into the wound and under the optimal hormonal influences, these cells proliferate. The endometrial tissue may have certain abilities that make implantation and transplantation possible during surgery. Inadvertent direct implantation during surgery is the most conceivable theory for incisional endometriosis. This implantation is subsequently stimulated by estrogen. Another theory is mainly based on the fact that peritoneal mesothelial cells undergo metaplasia to endometrial cells.

Surgical treatment of the parietal endometriosis required large extent of the excision, sometimes including the muscular and aponeurotic structures, this "sacrifice" is necessary for the removal of all the microscopic endometriotic sites, in order to prevent the recurrences. In large lesions, complete excision of the lesion may entail for closure after resection, the placement of a synthetic mesh or transfer of tissue. ${ }^{7}$ As ectopic endometrial tissue can theoretically undergo malignant transformation, histology evaluation is necessary. Malignant change of endometriosis in a cesarean scar is rare. Long standing recurrent scar endometriosis could undergo malignant changes and clinicians should be aware.

Only $21.3 \%$ of cases of malignant transformation of endometriosis occur at extragonadal pelvic sites, $4 \%$ of cases in scar after laparotomy.

The incidence of concomitant pelvic endometriosis with scar endometriosis has been reported to be ranging from $14.2 \%$ to $26 \% .^{8}$ All patients must be examined for concomitant pelvic endometriosis and post-operative follow up should be done for a couple of years.

Adjuvant hormonal therapy used after surgical excision decrease the recurrence rate from $42.9 \%$ to $11 \% .^{9-10}$ As it can address to those small foci that are undetectable, remain in place or could re-contaminate neighboring tissues by the use of the surgical maneuvers.

It is strong recommended that used sponge should be discarded immediately after cleaning the uterine cavity; the suture material used for uterus should not be reused while closing abdominal wall, and finally the surgical area should be cleaned thoroughly and irrigated with saline solution before closure.

\section{CONCLUSION}

A high index of suspicion of scar endometriosis is present whenever a women presents with a painful swelling in the abdominal scar, especially with a history of surgery.

Overall, general surgeons are infrequently involved in the management of cesarean section scar lesions. Thus, the lack of awareness makes the preoperative diagnosis unnoticed.

The only curative treatment is surgery, consisting in large excisions, in order to prevent recurrences. Large muscular masses may have to be excised, resulting in parietal defects that are solved by plastic surgery procedures.

Careful closure and avoidance of contamination following cesarean section may prevent scar endometriosis.

Funding: No funding sources Conflict of interest: None declared

Ethical approval: The study was approved by the Institutional Ethics Committee

\section{REFERENCES}

1. Wolf G, Singh K. Cesarean scar endometriosis: a review. Obstet Gynecol Surv. 1989;44:89-95.

2. Joly D, Harden H, Huguet C. Endometriosis of the abdominal pario in the long-term consequences of a cesarean. Lyon Chir. 1988;84(2):106-8. 
3. Blumenthal RD, Samoszuk M, Taylor AP, Brown G, Alisauskas R, Goldenberg DM. Degranulating eosinophils in human endometriosis, Am J Pathol. 2000;156(5):1581-8.

4. Botha AJ, Halliday AE, Flanagan JP, Endometriosis in gluteus muscle with surgical implantation. A case report. Acta Orthop Scand. 1991;62(5):497-9.

5. Ali T, Mohammed F, Hoford R, Maharaj D, Sookhoo S. Extrapelvic endometriosis presenting as unusual swellings of the buttock and thigh, west Indian. Med J. 2001;50(4):328-30.

6. Sirito R, Puppo A, Centurioni MG, Gustavino C. Incisional hernia on th $5-\mathrm{mm}$ trocar port site and subsequent wall endometriosis on the same site: a case report. Am J Obstet Gynecol. 2005;193(3pt 1):878-80.

7. Patterson GK, Winburn GP. Abdominal wall endometriomas: report of eight cases. Am J Surg. 1999;65:36-9.
8. Rani PR, Soundararaghavan S, Rajaram P. Endometriosis in abdominal scars - review of 27 cases. Int J Gynaecol Obstet. 1991;36:215-8.

9. Wang PH, Juang CM, Chao HT, Yu KJ, Yuan CC, $\mathrm{Ng}$ HT. Wound endometriosis: risk factor evaluation and treatment. J Chin Med Assoc. 2003;66(2):113-9.

10. Petakovic S, Perunicic P, Cuk D. Perineal endometriosis at the site of an episiotomy scar. Med Pregl. 1997;50(3-4):125-7.

Cite this article as: Juneja SK, Tandon P, Sharma A, Sharma A. Clinical profile and outcome of scar endometriosis in a tertiary care centre of Northern India. Int J Reprod Contracept Obstet Gynecol 2020;9:744-7. 